\title{
Robust Nonlinear EGR and VGT Control with Integral Action for Diesel Engines
}

\author{
Johan Wahlström* Lars Eriksson* \\ * Vehicular Systems, Linköping University, SWEDEN \\ Email: johwa@isy.liu.se
}

\begin{abstract}
A robust non-linear multivariable control design with integral action is proposed and investigated for control of EGR valve and VGT position in heavy duty diesel engines. The main control goal is to regulate oxygen/fuel ratio and intake manifold EGR-fraction. These are chosen as main performance variables since they are strongly coupled to the emissions. A recently developed non-linear control design based on feedback linearization is extended with integral action. The nonlinear controller gives an inner loop with good stability and robustness properties. It is shown that integral action is necessary to handle model errors so that the controller can track the performance variables specified in the outer loop. In particular the control design method utilizes a control Lyapunov function and inverse optimal control, which results in a control law with robustness properties interpretable as gain and phase margins. Furthermore, comparisons by simulation also show that the proposed control design successfully handles non-linear effects.
\end{abstract}

Keywords: Lyapunov function; inverse optimal control.

\section{INTRODUCTION}

Legislated emission limits for heavy duty trucks are constantly reduced. To fulfill the requirements, technologies like Exhaust Gas Recirculation (EGR) systems and Variable Geometry Turbochargers (VGT) have been introduced. The primary emission reduction mechanisms utilized to control the emissions are that $N O_{x}$ can be reduced by increasing the intake manifold EGR-fraction $x_{e g r}$ and smoke can be reduced by increasing the air/fuel ratio (Heywood, 1988). Note that exhaust gases, present in the intake, also contain oxygen which makes it more suitable to define and use the oxygen/fuel ratio $\lambda_{O}$ instead of the traditional air/fuel ratio. The main motive for this is that it is the oxygen contents that is crucial for smoke generation. Besides $\lambda_{O}$ it is natural to use EGR-fraction $x_{e g r}$ as the other main performance variable, but one could also use the burned gas fraction instead of the EGR-fraction.

The oxygen/fuel ratio $\lambda_{O}$ and EGR fraction $x_{e g r}$ depend in complicated ways on the EGR and VGT actuation. It is therefore necessary to have coordinated control of the EGR and VGT to reach the legislated emission limits in $N O_{x}$ and smoke. Various approaches for coordinated control of the EGR and VGT for emission abatement have been published. Guzzella and Amstutz (1998) presents a good overview of different control aspects of diesel engines with EGR and VGT, and in Nieuwstadt et al. (2000) there is a comparison of some control approaches with different selections of performance variables. Other control approaches are described in Amstutz and Re (1995), Nieuwstadt et al. (1998), Stefanopoulou et al. (2000), Amman et al. (2003), and Rückert et al. (2001).

A robust non-linear multivariable control design is proposed in Jankovic et al. (2000). This design includes con- struction of a Lyapunov function and inverse optimal control which provides a control law that handles interactions and non-linear properties in the system. However, in this design compressor mass flow $W_{c}$ and exhaust manifold pressure $p_{e m}$ are chosen as outputs and therefore the setpoints for $\lambda_{O}$ and $x_{e g r}$ are transformed to set-points for $W_{c}$ and $p_{e m}$. This transformation is based on a simplified third-order model, yielding model errors and consequently inconsistent set-points for $p_{e m}, W_{c}, \lambda_{O}$, and $x_{e g r}$ and therefore control errors, see Fig. 3. In order to decrease these control errors, this paper proposes a control design that extends the one in Jankovic et al. (2000) with integral action in Sec. 4. The aim is also to illustrate the advantages with integral action and non-linear control in Sec. 6. Further, Sec. 2 describes a mean value diesel engine model that is used in simulation for tuning and validation of the developed controller. The control design method used in this paper is described in Sec. 3. Finally, for efficient calibration an automatic controller tuning method is developed in Sec. 5.

\section{DIESEL ENGINE MODEL}

A model for a heavy duty diesel engine is used in simulation for tuning and validation of the developed controller. This diesel engine model is focused on the gas flows, see Fig. 1, and it is a mean value model with seven states: intake and exhaust manifold pressures $\left(p_{i m}\right.$ and $\left.p_{e m}\right)$, oxygen mass fraction in the intake and exhaust manifold ( $X_{\text {Oim }}$ and $\left.X_{\text {Oem }}\right)$, turbocharger speed $\left(\omega_{t}\right)$, and two states describing the actuator dynamics for the two control signals $\left(\tilde{u}_{e g r}\right.$ and $\left.\tilde{u}_{v g t}\right)$. These states are collected in a state vector $x$

$$
x=\left[\begin{array}{lllllll}
p_{\text {im }} & p_{\text {em }} & X_{\text {Oim }} & X_{\text {Oem }} & \omega_{t} & \tilde{u}_{\text {egr }} & \tilde{u}_{v g t}
\end{array}\right]^{T}
$$




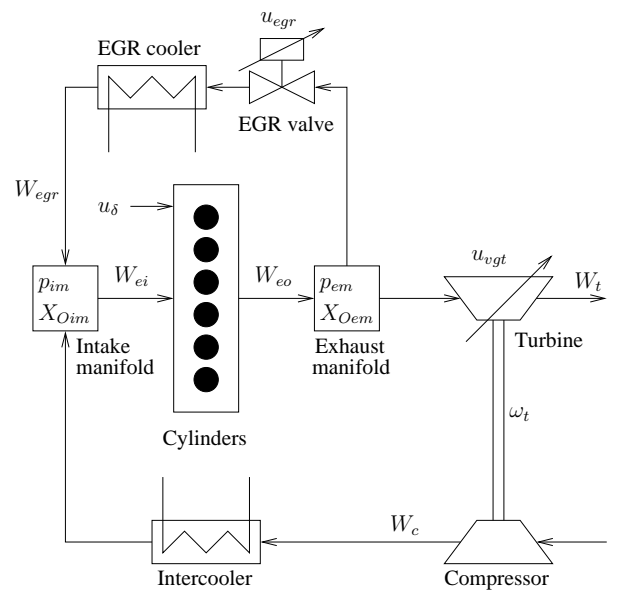

Fig. 1. Sketch of the diesel engine model used for simulation, control design, and tuning. It has five states related to the engine $\left(p_{i m}, p_{e m}, X_{\text {Oim }}, X_{\text {Oem }}\right.$, and $\left.\omega_{t}\right)$ and two for actuator dynamics $\left(\tilde{u}_{e g r}\right.$ and $\left.\tilde{u}_{v g t}\right)$.

There are no state equations for the manifold temperatures, since the pressures and the turbocharger speed govern the most important system properties, such as nonminimum phase behaviors, overshoots, and sign reversals, while the temperature states have only minor effects on these system properties.

The resulting model is expressed in state space form as

$$
\dot{x}=f\left(x, u, n_{e}\right)
$$

where the engine speed $n_{e}$ is considered as an input to the model, and $u$ is the control input vector

$$
u=\left[\begin{array}{lll}
u_{\delta} & u_{\text {egr }} & u_{v g t}
\end{array}\right]^{T}
$$

which contains mass of injected fuel $u_{\delta}$, EGR-valve position $u_{e g r}$, and VGT actuator position $u_{v g t}$.

A detailed description and derivation of the model together with a model tuning and a validation against test cell measurements is given in Wahlström (2006). The derivatives of the engine state variables are given by (1), and the oxygen concentration in the exhaust gas is calculated in (2). Further, the main performance variables are defined by $(3)$.

$$
\begin{aligned}
\frac{d}{d t} p_{e m}= & f_{1}(x, u), \quad \frac{d}{d t} \omega_{t}=f_{2}(x, u) \\
\frac{d}{d t} p_{i m}= & \frac{R_{a} T_{i m}}{V_{i m}}\left(W_{c}+W_{e g r}-W_{e i}\right) \\
\frac{d}{d t} X_{O i m}= & \frac{R_{a} T_{i m}}{p_{i m} V_{i m}}\left(\left(X_{O e m}-X_{O i m}\right) W_{e g r}+\right. \\
& \left.\left(X_{O c}-X_{O i m}\right) W_{c}\right) \\
\frac{d}{d t} X_{O e m}= & \frac{R_{e} T_{e m}}{p_{e m} V_{e m}}\left(X_{O e}-X_{O e m}\right)\left(W_{f}+W_{e i}\right) \\
X_{O e}=\frac{W_{e i} X_{O i m}-W_{f}(O / F)_{s}}{W_{f}+W_{e i}} & W_{e g r}, \quad \lambda_{O}=\frac{W_{e i} X_{O i m}}{W_{f}(O / F)_{s}} \\
x_{e g r}= & \frac{W_{c}+W_{e g r}}{W_{\text {Oen }}}
\end{aligned}
$$

\section{ROBUST NONLINEAR CONTROL}

The control design method used in this paper is based on a non-linear multivariable method proposed in Sepul- chre et al. (1997) and Jankovic et al. (1999). It includes construction of a Control Lyapunov Function (CLF) and inverse optimal control that guarantees robustness of optimal controllers. The control design method is briefly reviewed below.

In inverse optimal control, the goal is to first design a control law and then determine which cost function it minimizes. However, in order to obtain a simple relation between the control law and the cost function, the cost function is chosen quadratic in $u$ according to

$$
\int_{0}^{\infty} l(x)+u^{T} R(x) u d t, \quad l(x)>0, \quad R(x)>0
$$

In standard optimal control (Bryson and Ho, 1975), the control law that minimizes (4) for the system

$$
\begin{aligned}
& \dot{x}=f(x)+g(x) u \\
& y=h(x)+j(x) u, \quad x \in \mathbb{R}^{n}, \quad u, y \in \mathbb{R}^{m}
\end{aligned}
$$

is found by solving the Hamilton-Jacobi-Bellman equation

$$
0=\min _{u}\left[l(x)+u^{T} R(x) u+L_{f} V(x)+L_{g} V(x) u\right]
$$

that has the solution

$$
u(x)=-\frac{1}{2} R^{-1}\left(L_{g} V\right)^{T}(x)
$$

where $V(x)$ is a Control Lyapunov Function (CLF) and the notation $L_{q} V(x)$ denotes the Lie derivate of $V(x)$ along the vector field $q(x)$. Consequently, the goal is to first design $V(x)$ and the matrix $R(x)$, and then determine $l(x)$ in order to see which cost function the control law (6) minimizes.

The optimal control law (6) guarantees stability and robustness properties. In particular, if

$$
R^{-1}=\operatorname{diag}\left\{\gamma_{1}, \cdots, \gamma_{m}\right\}
$$

and

$$
\gamma_{i}>2 \max _{x} p(x)
$$

where

$$
p(x)= \begin{cases}\frac{L_{f} V+\sqrt{\left(L_{f} V\right)^{2}+\left\|L_{g} V\right\|^{4}}}{\left\|L_{g} V\right\|^{2}} & \text { if }\left\|L_{g} V\right\| \neq 0 \\ 0 & \text { if }\left\|L_{g} V\right\|=0\end{cases}
$$

then the control law (6) gives asymptotic stability and it is robust to input uncertainties that have $(1 / 2, \infty)$ gain margin and $\pm 60^{\circ}$ phase margin (Jankovic et al., 2000).

\section{CONTROL DESIGN WITH INTEGRAL ACTION}

A control design without integral action of a diesel engine using the method in Sec. 3 is proposed in Jankovic et al. (2000). This gives an inner loop that handles nonlinearities and decouples the system, in addition it has good stability and robustness properties. However, as will be shown in Sec. 6, integral action is necessary to handle model errors so that the controller can track the performance variables $\lambda_{O}$ and $x_{e g r}$ specified in an outer loop. Therefore, the proposed design is extended with integral action, resulting in the proposed closed-loop system with integral action shown in Fig. 2.

\subsection{Control design model}

In order to get a simple control law, the seventh-order model in Sec. 2 is simplified to a model with three states: 


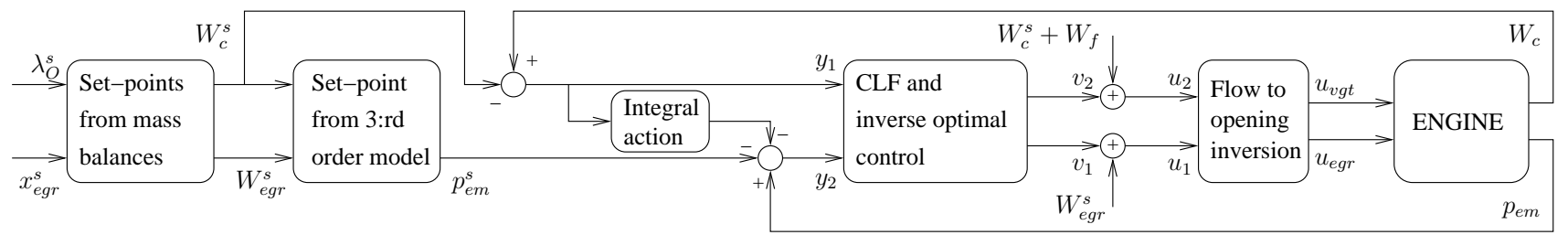

Fig. 2. Block diagram of the closed-loop system, showing; set-points calculations, integral action on the flow $W_{c}$, nonlinear control of the flows $u_{1}$ and $u_{2}$, and flow to opening inversion.

$p_{i m}, p_{e m}$, and the compressor power $P_{c}$. This model is based on the control design model developed in Jankovic et al. (2000).

$$
\begin{aligned}
\dot{p}_{i m} & =k_{i m}\left(W_{c}+u_{1}-k_{e} p_{i m}\right) \\
\dot{p}_{e m} & =k_{e m}\left(k_{e} p_{i m}-u_{1}-u_{2}+W_{f}\right) \\
\dot{P}_{c} & =\frac{1}{\tau}\left(\eta_{m} P_{t}-P_{c}\right) \\
W_{c} & =\frac{\eta_{c} P_{c}}{T_{a m b} c_{p a}\left(\left(p_{i m} / p_{a m b}\right)^{\mu_{a}}-1\right)} \\
P_{t}= & \eta_{t} c_{p e} T_{e m}\left(1-\left(p_{a m b} / p_{e m}\right)^{\mu_{e}}\right) u_{2}
\end{aligned}
$$

The variables $k_{e m}=k_{e m}\left(T_{e m}\right), W_{f}=W_{f}\left(u_{\delta}, n_{e}\right)$, and $k_{e}=k_{e}\left(n_{e}\right)$ are treated as external slowly varying signals and $k_{i m}, \tau, \eta_{m}, \eta_{c}, T_{a m b}, c_{p a}, p_{a m b}, \mu_{a}, \eta_{t}, c_{p e}$, and $\mu_{e}$ are constants.

\subsection{Outputs and set-points}

The design objective is to regulate $\lambda_{O}$ and $x_{\text {egr }}$ to their set-points $\lambda_{O}^{s}$ and $x_{e g r}^{s}$. However, $\lambda_{O}$ can not be calculated from the control design model in Sec. 4.1. Further, $p_{\text {em }}$ has to be chosen as one output in order to get stable zero dynamics (Jankovic et al., 2000). Therefore, the following outputs are chosen

$$
y_{1}=W_{c}-W_{c}^{s}, \quad y_{2}=p_{e m}-p_{e m}^{s}
$$

The set-points $\lambda_{O}^{s}$ and $x_{e g r}^{s}$ are transformed to the setpoints $W_{c}^{s}$ and $p_{e m}^{s}$ in two steps. Firstly, the equilibriums for $W_{c}$ and $W_{\text {egr }}$ of the mass balances (1b)-(1d) are calculated from $\lambda_{O}^{s}$ and $x_{e g r}^{s}$

$$
\begin{aligned}
& W_{c}^{s}=\frac{W_{f}}{2 X_{O c}}\left(\beta+\sqrt{\beta^{2}+4 \lambda_{O}^{s}(O / F)_{s}\left(1-x_{e g r}^{s}\right) X_{O c}}\right) \\
& W_{e g r}^{s}=\frac{x_{e g r}^{s}}{1-x_{e g r}^{s}} W_{c}^{s}
\end{aligned}
$$

where

$$
\beta=\left(\lambda_{O}^{s}(O / F)_{s}-X_{O c}\right)\left(1-x_{e g r}^{s}\right)+(O / F)_{s} x_{e g r}^{s},
$$

$X_{O c}$ is the constant oxygen concentration in air passing the compressor, and $(O / F)_{s}$ is the stoichiometric relation between oxygen and fuel masses. Secondly, the equilibriums for $p_{i m}$ and $p_{e m}$ of the third-order model in Sec. 4.1 are calculated from $W_{c}^{s}$ and $W_{e q r}^{s}$

$$
\begin{aligned}
p_{i m}^{s} & =\frac{W_{c}^{s}+W_{e g r}^{s}}{k_{e}} \\
p_{e m}^{s} & =p_{a m b}\left(1-\frac{c_{p a}\left(\left(\frac{p_{i m}^{s}}{p_{a m b}}\right)^{\mu_{a}}-1\right) T_{a m b} W_{c}^{s}}{c_{p e} \eta_{c m t} T_{e m}\left(W_{c}^{s}+W_{f}\right)}\right)^{-\frac{1}{\mu_{e}}}
\end{aligned}
$$

\subsection{Integral action}

Since the set-points (11) are based on the simplified thirdorder model, model errors lead to that the values for $p_{i m}^{s}$ and $p_{e m}^{s}$ are inconsistent with the set-points for $W_{c}$ and $W_{e g r}$ in (10). As a consequence, control errors will appear in (9), yielding errors in $\lambda_{O}$ and $x_{e g r}$ (this will be illustrated in Fig. 3). In order to decrease these control errors, the following approximate integral action is used

$$
\dot{e}=-\delta e-K\left(W_{c}-W_{c}^{s}\right)=-\delta e-K y_{1}
$$

where $\delta$ is small, but must be positive to ensure stable zero dynamics. The choice of $y_{1}$ as input to the integral action ensures that the set-point $W_{c}^{s}$ is achieved. The next step is then to ensure that $W_{e g r}^{s}$ is achieved and this is done by feeding the state $e$ into $p_{e m}^{s}$. The set-point $p_{e m}^{s}$ depends nonlinearly on $W_{c}^{s}$ and $W_{e g r}^{s}$ it is therefore natural to utilize (11) when determining the gain from $e$ to $p_{e m}^{s}$. As a result the following set-point for $p_{\text {em }}$ is received

$$
\begin{aligned}
& \tilde{p}_{i m}^{s}(e)=\frac{W_{c}^{s}+W_{e g r}^{s}+e}{k_{e}} \\
& \tilde{p}_{e m}^{s}(e)=p_{a m b} \cdot \\
& \left(1-\frac{c_{p a}\left(\left(\frac{\tilde{p}_{i m}^{s}(e)}{p_{a m b}}\right)^{\mu_{a}}-1\right) T_{a m b}\left(W_{c}^{s}+e\right)}{c_{p e} \eta_{c m t} T_{e m}\left(W_{c}^{s}+W_{f}+e\right)}\right)^{-\frac{1}{\mu_{e}}}
\end{aligned}
$$

To simplify the control design, the Taylor expansion of $\tilde{p}_{e m}^{s}(e)$ is used according to

$$
\tilde{p}_{\text {em }}^{s}(e) \approx \tilde{p}_{\text {em }}^{s}(0)+\frac{d}{d e} \tilde{p}_{\text {em }}^{s}(0) e=p_{\text {em }}^{s}+\frac{d}{d e} \tilde{p}_{e m}^{s}(0) e
$$

where $\frac{d}{d e} \tilde{p}_{e m}^{s}(0)=d_{1} / d_{2}$ with

$$
\begin{aligned}
& d_{1}=\left[\mu_{a} W_{c}^{s}\left(W_{c}^{s}+W_{f}\right)\left(W_{c}^{s}+W_{e g r}^{s}\right)^{\mu_{a}-1}+\right. \\
& \left.\left(\left(W_{c}^{s}+W_{e g r}^{s}\right)^{\mu_{a}}-k_{e}^{\mu_{a}} p_{a m b}{ }^{\mu_{a}}\right) W_{f}\right] c_{p a}\left(p_{e m}^{s}\right)^{\mu_{e}+1} T_{a m b} \\
& d_{2}=c_{p e} \eta_{c m t} k_{e}{ }^{\mu_{a}} \mu_{e} p_{a m b}{ }^{\mu_{a}+\mu_{e}} T_{e m}\left(W_{c}^{s}+W_{f}\right)^{2}
\end{aligned}
$$

Using the set-point (13) for $p_{e m}$, the outputs become

$$
y_{1}=W_{c}-W_{c}^{s}, \quad y_{2}=p_{e m}-p_{e m}^{s}-d_{1} e / d_{2}
$$

Note that integral action can not be performed on $\lambda_{O}$ since $\lambda_{O}$ can not be calculated from the control design model. Further, if integral action is performed on $x_{\text {egr }}$, stable zero dynamics can not be guaranteed.

\subsection{Feedback linearization}

The first step in the control design method is to construct a CLF $V(x)$, which is done using feedback linearization. For the fourth order model (8) and (12), and the outputs (14), the relative degrees become $r_{1}=1$ and $r_{2}=1$. Consequently, $\dot{y}$ can be formulated as

$$
\dot{y}=G(x) u+F(x)
$$

where $\eta_{c m t}=\eta_{c} \eta_{m} \eta_{t}$. 
where the matrix

$$
G(x)=\left[\begin{array}{cc}
-a & b \\
-k_{e m} & -k_{e m}
\end{array}\right]
$$

is invertible with

$$
\begin{gathered}
a=\frac{k_{i m} \mu_{a}\left(\frac{p_{i m}}{p_{a m b}}\right)^{\mu_{a}-1} W_{c}}{p_{a m b}\left(\left(\frac{p_{i m}}{p_{a m b}}\right)^{\mu_{a}}-1\right)} \\
b=\frac{c_{p e} \eta_{c m t}\left(1-\left(\frac{p_{a m b}}{p_{e m}}\right)^{\mu_{e}}\right) T_{e m}}{c_{p a}\left(\left(\frac{p_{i m}}{p_{a m b}}\right)^{\mu_{a}}-1\right) \tau T_{a m b}}
\end{gathered}
$$

and where

$$
F(x)=\left[\begin{array}{c}
a k_{e} p_{i m}-a W_{c}-\frac{W_{c}}{\tau} \\
\frac{d_{1}\left(\delta e+K\left(W_{c}-W_{c}^{s}\right)\right)}{d_{2}}+k_{e m}\left(k_{e} p_{i m}+W_{f}\right)
\end{array}\right]
$$

Then by applying the feedback and input transformation

$$
u=G^{-1}(x)(-\alpha y-F(x)+w)
$$

and the change of coordinates $x \rightarrow[y, z]$, the system (8) and (12) is transformed into the system

$$
\begin{aligned}
& \dot{y}=-\alpha y+w \\
& \dot{z}=f_{0}(y, z)+g_{0}(y, z) w
\end{aligned}
$$

where $w$ is the new input, $\alpha$ is a positive scalar constant, and $z=\left[p_{i m}, e\right]^{T}$.

\subsection{Stability of the zero dynamics}

When feedback linearization is used it is necessary to investigate the stability of the zero dynamics, which are defined by

$$
\dot{z}=f_{0}(0, z)
$$

For (18) the zero dynamics become

$$
\begin{aligned}
\dot{p}_{i m} & =\frac{q_{1}\left(p_{i m}\right)}{q_{2}\left(p_{i m}, e\right)}\left(p_{i m}^{\mu_{a}}-q_{3}(e)\right) \\
\dot{e} & =-\delta e
\end{aligned}
$$

where

$$
\begin{aligned}
& q_{1}\left(p_{i m}\right)=c_{p a} k_{i m} p_{i m} T_{a m b} W_{c}^{s} \\
& q_{2}\left(p_{i m}, e\right)=-c_{p a} k_{i m} \mu_{a} p_{i m}{ }^{\mu} T_{a m b} \tau W_{c}^{s}- \\
& c_{p e} \eta_{c m t} p_{a m b} \mu_{a}\left(1-\left(\frac{p_{a m b}}{\frac{d_{1} e}{d_{2}}+p_{e m}^{s}}\right)^{\mu_{e}}\right) p_{i m} T_{e m} \\
& q_{3}(e)=\frac{p_{a m b}{ }^{\mu_{a}}}{c_{p a} k_{e m} T_{a m b} W_{c}^{s}}\left(\left(\frac{d_{1} \delta e}{d_{2}}+k_{e m}\left(W_{c}^{s}+W_{f}\right)\right) .\right. \\
& \left.\left(1-\left(\frac{p_{a m b}}{\frac{d_{1} e}{d_{2}}+p_{e m}^{s}}\right)^{\mu_{e}}\right) c_{p e} \eta_{c m t} T_{e m}+c_{p a} k_{e m} T_{a m b} W_{c}^{s}\right)
\end{aligned}
$$

To analyze the stability of the zero dynamics (19), the Lyapunov function

$$
V=\frac{1}{2} c_{z 1} z_{1}^{2}+\frac{1}{2} c_{e} e^{2}
$$

is used where $z_{1}=p_{i m}^{\mu_{a}}-q_{3}(e)$. The zero dynamics (19) are asymptotically stable if

$$
\begin{aligned}
\dot{V}= & c_{z 1} z_{1}^{2}\left(\mu_{a} p_{i m}^{\mu_{a}-1} \frac{q_{1}}{q_{2}}+\frac{\delta c_{z 1}\left(q_{3}^{\prime}(e)\right)^{2}}{4 c_{e}}\right)- \\
& c_{e} \delta\left(e-\frac{c_{z 1}}{2 c_{e}} q_{3}^{\prime}(e) z_{1}\right)^{2}<0
\end{aligned}
$$

for all $\left[z_{1}, e\right] \neq 0$ which is true if $\delta>0$ and

$$
\frac{c_{z 1}}{c_{e}}<-\frac{4}{\delta\left(q_{3}^{\prime}(e)\right)^{2}} \mu_{a} p_{i m}^{\mu_{a}-1} \frac{q_{1}}{q_{2}}
$$

This is possible if $q_{1} / q_{2}<0$, which is true if $d_{1} e / d_{2}+$ $p_{e m}^{s} \geq p_{a m b}$. This relation is achieved by implementing the integral action (12) in discrete form with anti-windup according to

$$
\begin{aligned}
& \epsilon_{n}=e_{n-1}-T_{s}\left(\delta e_{n-1}+K y_{1}\right) \\
& e_{n}= \begin{cases}\epsilon_{n}, & \text { if } \epsilon_{n} \geq e_{\min } \\
e_{\min } & , \text { if } \epsilon_{n}<e_{\min }\end{cases}
\end{aligned}
$$

where $T_{s}$ is the sample time and

$$
e_{m i n}=-\frac{d_{2}}{d_{1}}\left(p_{e m}^{s}-p_{a m b}\right)
$$

\subsection{Construction of a CLF}

In order to obtain a simple control law, a quadratic Lyapunov function

$$
V=c_{1} y_{1}^{2}+c_{2} y_{2}^{2}
$$

is constructed, which is a CLF for the system (17), since

$$
\dot{V}=-2 c_{1} \alpha y_{1}^{2}-2 c_{2} \alpha y_{2}^{2}<0
$$

for $w=0$ and all $y \neq 0$.

\subsection{Control law}

Using the CLF (20), the control law (6) can be applied to the system (17). However, this control law will contain the non-linear cancellation (16) that is sensitive to model errors. In order to obtain a robust control law, this cancellation is avoided by applying the control law (6) to the system (15) instead, using the same Lyapunov function $V$. Since $V$ is a CLF for the system (17), it is a CLF for all systems that can be obtained from (17) by feedback, coordinate, and input transformations which includes the system (15).

To apply the control law (6), the control inputs are centered at their set-point values

$$
v_{1}=u_{1}-W_{e g r}^{s}, \quad v_{2}=u_{2}-W_{c}^{s}-W_{f}
$$

This leads to that $v=0$ renders the equilibrium point $y=0$.

A simple control law for the system (15) is achieved by choosing $R^{-1}=\operatorname{diag}\left\{\gamma_{1}, \gamma_{2}\right\}$, yielding

$$
\left[\begin{array}{l}
v_{1} \\
v_{2}
\end{array}\right]=-\frac{1}{2} R^{-1}\left(L_{G} V\right)^{T}=\left[\begin{array}{c}
\gamma_{1}\left(c_{1} a y_{1}+c_{2} k_{e m} y_{2}\right) \\
\gamma_{2}\left(-c_{1} b y_{1}+c_{2} k_{e m} y_{2}\right)
\end{array}\right]
$$

This concludes the description of the control system in Fig. 2.

\section{AUTOMATIC CONTROLLER TUNING}

In the proposed control design there are five tuning parameters: $\gamma_{1}, \gamma_{2}, c_{1}, c_{2}$, and $K$. The tuning objectives are to minimize the error between $\lambda_{O}$ and $\lambda_{O}^{s}$ and between $x_{e g r}$ and $x_{e g r}^{s}$, and to achieve the inequality (7). However, it is difficult to achieve these objectives by manually tuning, especially the last objective. Therefore an efficient method for automatic tuning of the parameters is developed. 


\subsection{Cost function for tuning}

The automatic tuning method is obtained by formulating a non-linear least squares problem

$$
\begin{aligned}
& \min V(\theta) \\
& \theta>0
\end{aligned}
$$

where $\theta$ is the tuning parameters

$$
\theta=\left[\gamma_{1}, \gamma_{2}, c_{1}, c_{2}, K\right]^{T}
$$

The cost function $V(\theta)$ is calculated as

$$
\begin{aligned}
& V(\theta)=\sum_{i=1}^{N} \gamma_{\lambda_{O}}\left(\frac{e_{\lambda_{O}}\left(t_{i}\right)}{\lambda_{\text {ONorm }}}\right)^{2}+\gamma_{\text {egr }}\left(\frac{e_{\text {xegr }}\left(t_{i}\right)}{x_{\text {egr Norm }}}\right)^{2} \\
& +\left(\frac{u_{\text {egr }}\left(t_{i}\right)-u_{\text {egr }}\left(t_{i-1}\right)}{u_{\text {egr Norm }}}\right)^{2}+\left(\frac{u_{\text {vgt }}\left(t_{i}\right)-u_{\text {vgt }}\left(t_{i-1}\right)}{u_{\text {vgt Norm }}}\right)^{2} \\
& +\gamma_{p}\left(\frac{\max \left(2 p\left(x\left(t_{i}\right)\right) / \min \left(\gamma_{1}, \gamma_{2}\right)-1,0\right)}{p_{\text {Norm }}}\right)^{2}
\end{aligned}
$$

where $t_{i}$ is the time at sample number $i$. The motives for the different terms in the cost function are:

Term 1: Minimizes $\lambda_{O}$ error $\left(e_{\lambda_{O}}=\lambda_{O}^{s}-\lambda_{O}\right)$

Term 2: Minimizes EGR error $\left(e_{x e g r}=x_{e g r}^{s}-x_{e g r}\right)$.

Term 3 and 4: Minimize oscillations in the EGR valve and VGT control signals. The terms have equal weight.

Term 5: If the inequality (7) is not satisfied, this term minimizes the difference between the right and left hand side of (7) using a high penalty, $\gamma_{p}=100$. However, this does not guarantee that the inequality (7) is satisfied for all operating points.

As seen in (22) all the terms are normalized in order to get the same order of magnitude for the five terms. The weighting factors $\gamma_{\lambda_{O}}$ and $\gamma_{e g r}$ are set to 1 and the constant $\delta$ is set to $10^{-6}$.

\subsection{Optimization}

A solver has been developed for the optimization problem stated in the previous section, and it consists of three phases. Firstly, the tuning parameters are initialized manually. Secondly, a globalization heuristic method is used to scan a large region around the initial values in order to avoid ending up in a bad local minimum. Thirdly, a standard non-linear local least squares solver is used. A detailed description of the heuristic method is given in Wahlström (2006).

\section{CONTROLLER EVALUATION}

The performance of the control system in Fig. 2 is evaluated by comparing three different control systems:

CLF with integral action: The control system in Fig. 2 where the controller tuning method in Sec. 5 is applied to the transient cycle defined by Tab. 1 .

CLF without integral action: Same as above, but $K$ is set to zero and removed from $\theta$ in (21).

PID: The control structure with PID controllers and automatic tuning method proposed in Wahlström (2006). It has the following main feedback loops

$$
\begin{aligned}
& u_{\text {egr }}=-\mathrm{PID}_{1}\left(e_{\lambda_{O}}\right) \\
& u_{v g t}=-\mathrm{PID}_{2}\left(e_{\text {xegr }}\right)
\end{aligned}
$$

Table 1. A definition of the transient cycle that is used for tuning and consists of steps between 17 different operating points. The cycle starts at 1 and ends at 17, and spends $10 \mathrm{~s}$ in each point. The values for $\lambda_{O}^{s}$ and $x_{e g r}^{s}$ are obtained by calculating the stationary points of the seventh order model for the 17 points below.

\begin{tabular}{c|c||c|c|c|c|c|}
$u_{\delta}[\mathrm{mg} /$ cycle $]$ & $u_{\text {egr }}[\%]$ & \multicolumn{5}{|c|}{ Operating points } \\
\hline \hline \multirow{2}{*}{230} & 40 & 6 & 7 & & 11 & 12 \\
\cline { 2 - 7 } & 10 & 5 & 8 & & 10 & 9 \\
\hline \multirow{2}{*}{60} & 25 & & & 17 & & \\
\hline \hline \multirow{2}{*}{60} & 40 & 4 & 3 & & 14 & 13 \\
\cline { 2 - 7 } & 10 & 1 & 2 & & 15 & 16 \\
\hline & $u_{v g t}[\%]$ & 30 & 60 & 45 & 30 & 60 \\
\cline { 2 - 7 } & $n_{e}[\mathrm{rpm}]$ & \multicolumn{2}{|c||}{1000} & 1500 & \multicolumn{2}{|c|}{2000}
\end{tabular}
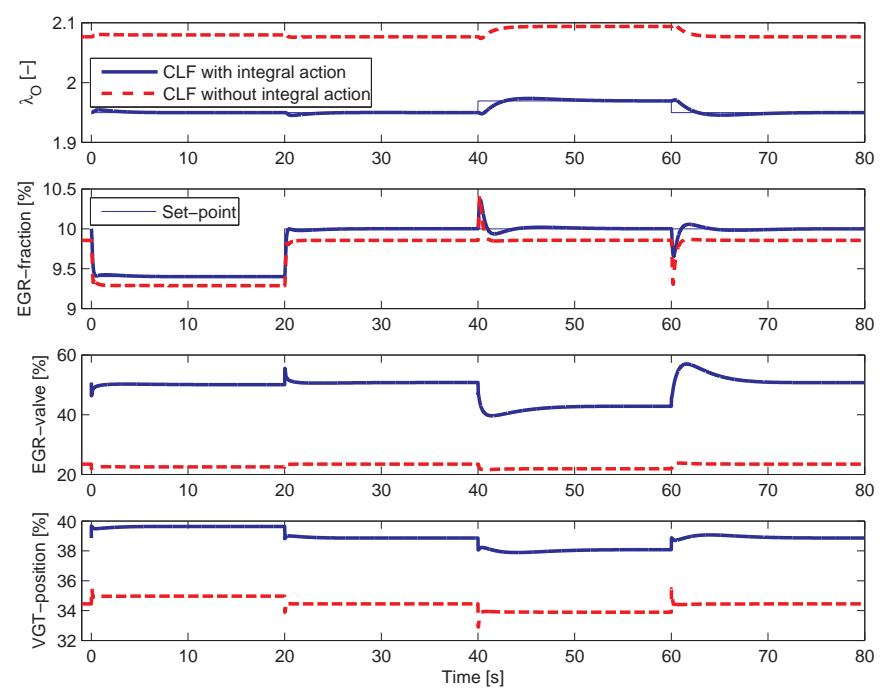

Fig. 3. Illustrative example simulating CLF with and without integral action showing that the control errors are reduced if an integral action is used.

where $e_{\lambda_{O}}=\lambda_{O}^{s}-\lambda_{O}$ and $e_{x e g r}=x_{e g r}^{s}-x_{e g r}$.

The full seventh order model, described in Sec. 2, is used as plant model to evaluate the three control systems above. All their control parameters are held constant for the entire operating region, i.e. no gain scheduling is used. In addition, a low pass filter is applied to all variables assumed to come from an observer.

\subsection{Benefits with integral action}

In Fig. 3, "CLF with integral action" and "CLF without integral action" are simulated. Steps in $\lambda_{O}^{s}$ and $x_{e g r}^{s}$ are performed at $n_{e}=1200 \mathrm{rpm}$ and $u_{\delta}=130 \mathrm{mg} /$ cycle. The result is that the control errors in $\lambda_{O}$ and $x_{e g r}$ are reduced if an integral action is used.

\subsection{Benefits with non-linear control}

In Fig. 4, "CLF with integral action" and PID are simulated. The same steps in $\lambda_{O}^{s}$ and $x_{e g r}^{s}$ are performed as in Fig. 3. The result is that PID gives slower control compared to CLF.

In Fig. 5, "CLF with integral action" and PID are simulated at two other steps in $\lambda_{O}^{s}$ and $x_{e g r}^{s}$ compared to Fig. 4. 

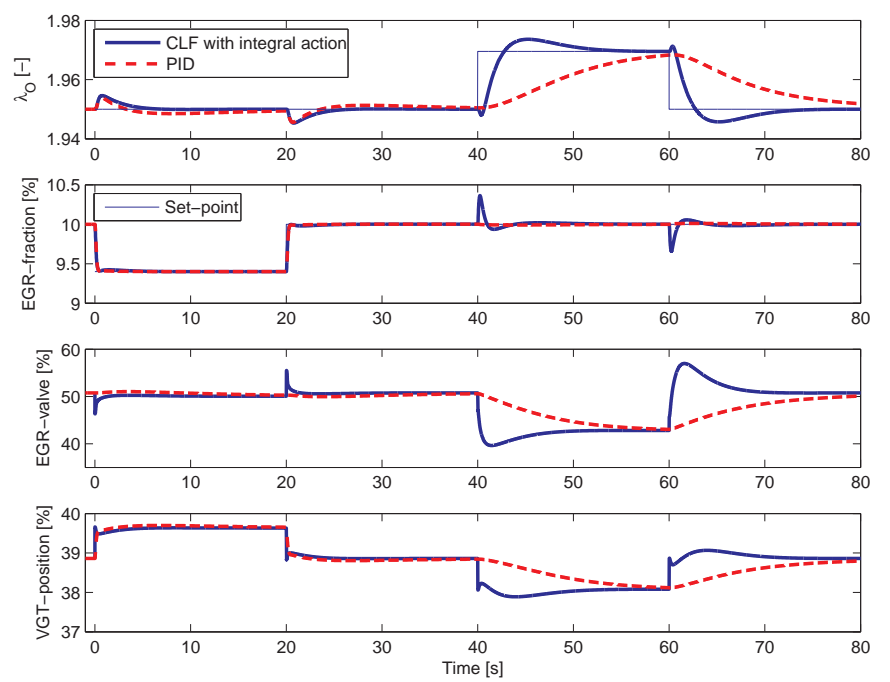

Fig. 4. Illustrative example simulating CLF with integral action and PID showing that PID gives slower control compared to CLF.
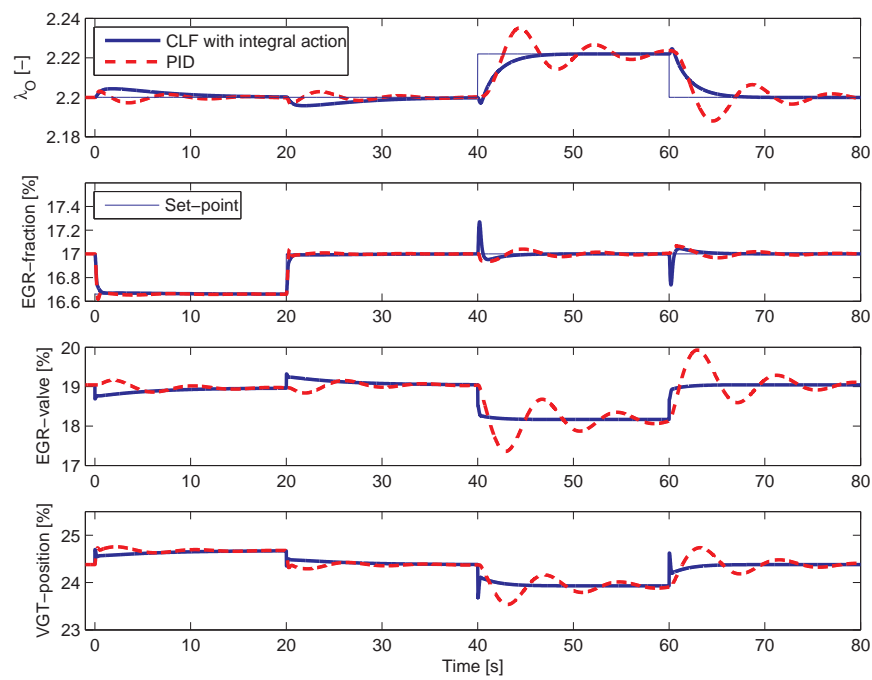

Fig. 5. Illustrative example simulating CLF with integral action and PID at two other steps in $\lambda_{O}^{s}$ and $x_{e g r}^{s}$ compared to Fig. 4 showing that PID gives oscillations and CLF gives no oscillations.

The operating point in Fig. 5 renders higher DC-gains in $u_{e g r} \rightarrow \lambda_{O}$ and $u_{v g t} \rightarrow x_{\text {egr }}$ (the loops that are used as feedbacks in (23)) compared to the operating point in Fig. 4. The result is that PID gives oscillations, while CLF gives no oscillations. Since the same control parameters are used in Fig. 4 and 5 for CLF respectively PID, the conclusion is that CLF handles the non-linear effects in the diesel engine, such as non-linear gains.

\section{CONCLUSIONS}

A robust non-linear multivariable control design with integral action has been proposed and investigated for control of oxygen/fuel ratio and EGR fraction. The control design method includes construction of a CLF by using exact linearization and inverse optimal control, which provides a control law that is robust to static input uncertainties that have $(1 / 2, \infty)$ gain margin and $\pm 60^{\circ}$ phase margin. This method is applied to a fourth order model including integral action on the compressor mass flow.

Further, simulations of the proposed control design show that control errors are reduced if an integral action is used compared to a control design without integral action. The simulations also show that the proposed control design handles the non-linear effects in the diesel engine.

\section{ACKNOWLEDGEMENTS}

The Swedish Energy Agency and Scania CV AB are gratefully acknowledged for their support.

\section{REFERENCES}

M. Amman, N.P. Fekete, L. Guzzella, and A.H. Glattfelder. Model-based Control of the VGT and EGR in a Turbocharged Common-Rail Diesel Engine: Theory and Passenger Car Implementation. SAE Technical paper 2003-01-035\%, January 2003.

A. Amstutz and L. Del Re. EGO sensor based robust output control of EGR in diesel engines. IEEE Transactions on Control System Technology, pages 37-48, 1995.

Arthur E. Bryson and Yu-Chi Ho. Applied Optimal Control. Taylor and Franchis, 1975.

L. Guzzella and A. Amstutz. Control of diesel engines. IEEE Control Systems Magazine, 18:53-71, 1998.

J.B. Heywood. Internal Combustion Engine Fundamentals. McGraw-Hill Book Co, 1988.

M. Jankovic, M. Jankovic, and I.V. Kolmanovsky. Constructive lyapunov control design for turbocharged diesel engines. IEEE Transactions on Control Systems Technology, 2000.

Mrdjan Jankovic, Rodolphe Sepulchre, and Petar Kokotovic. CLF based designs with robustness to dynamic input uncertainties. Systems and Control Letters, 1999.

M. Nieuwstadt, P.E. Moraal, I.V. Kolmanovsky, A. Stefanopoulou, P. Wood, and M. Widdle. Decentralized and multivariable designs for EGR-VGT control of a diesel engine. In IFAC Workshop, Advances in Automotive Control, 1998.

M.J. Nieuwstadt, I.V. Kolmanovsky, P.E. Moraal, A.G. Stefanopoulou, and M. Jankovic. EGR-VGT control schemes: Experimental comparison for a high-speed diesel engine. IEEE Control Systems Magazine, 2000.

J. Rückert, A. Schloßer, H. Rake, B. Kinoo, M. Krüger, and S. Pischinger. Model based boost pressure and exhaust gas recirculation rate control for a diesel engine with variable turbine geometry. In IFAC Workshop: Advances in Automotive Control, 2001.

Rodolphe Sepulchre, Mrdjan Jankovic, and Petar Kokotovic. Constructive Nonlinear Control. Springer-Verlag, 1997.

A.G. Stefanopoulou, I.V. Kolmanovsky, and J.S. Freudenberg. Control of variable geometry turbocharged diesel engines for reduced emissions. IEEE Transactions on Control Systems Technology, 8(4), July 2000.

Johan Wahlström. Control of EGR and VGT for emission control and pumping work minimization in diesel engines. Technical report, Linköping University, 2006. 